\title{
SRJ09, a promising anticancer drug lead: elucidation of mechanisms of antiproliferative and apoptogenic effects and assessment of in vivo antitumor efficacy
}

\begin{abstract}
SRJ09 (3,19-(2-bromobenzylidene)andrographolide), a semisynthetic andrographolide (AGP) derivative, was shown to induce $\mathrm{G}_{1}$ cell cycle arrest and eventually apoptosis in breast and colon cancer cell lines. The present investigation was carried out to elucidate the mechanisms cell cycle arrest and apoptosis and evaluate the in vivo antitumor activity of SRJ09. The in vitro growth inhibitory properties of compounds were assessed in colon (HCT-116) and breast (MCF-7) cancer cell lines. Immunoblotting was utilized to quantitate the protein levels in cells. The gene expressions were determined using reverse transcriptase PCR (RT-PCR). Pharmacokinetic investigation was carried out by determining SRJ09 levels in plasma of Balb/C mice using HPLC. In vivo antitumor activity was evaluated in athymic mice carrying HCT-116 colon tumor xenografts. SRJ09 displayed improved in vitro activity when compared with AGP by producing rapid cell killing effect in vitro. Its activity was not compromised in MES-SA/Dx5 multidrug resistant (MDR) cells expressing p-glycoprotein. Cells treated with SRJ09 $(0.1 \square 10 \mu \mathrm{M})$ displayed increased p21 protein level, which corresponded with gene expression. Whereas CDK4 protein level and gene expression was suppressed. The treatment did not affect cyclin D1. Changes of these proteins paralleled $\mathrm{G}_{1}$ cell cycle arrest in both cell lines as determined by flow cytometry. Induction of apoptosis by SRJ09 in HCT-116 cells which occurred independent of p53 and bcl-2 was inhibited in the presence of caspase 8 inhibitor, implicating the extrinsic apoptotic pathway. A single dose $(100 \mathrm{mg} / \mathrm{kg}$, i.p) of SRJ09 produced a plasma concentration range of $12 \square 30.4 \mu \mathrm{M}$. At $400 \mathrm{mg} / \mathrm{kg}$ (q4dX3), it significantly retarded growth of tumor xenografts. The antitumor activity of SRJ09 is suggested mediated via the induction of p21 expression and suppression of CDK-4 expression without affecting cyclin D1 to trigger $\mathrm{G}_{1}$ arrest leading to apoptosis.
\end{abstract}

Keyword: Andrographolide derivatives; p21; CDK-4; Apoptosis; Cell cycle arrest; Pharmacokinetics 